\title{
An Enhanced Detection System for Elderly Person Monitoring using Embedded System
}

\author{
Bhavadharani $\mathrm{C}^{\mathbf{1}}$, Arumuga Selvi $\mathrm{S}^{2}$, Divya $\mathrm{S}^{3}$, Logapriya $\mathrm{N}^{4}$ \\ UG Student, Department of ECE, Unnamalai Institute of Technology, Tamilnadu, India ${ }^{1,2,3,4}$
}

\begin{abstract}
The main focus of the paper is to implement a prototype model for the real time elderly person monitoring and fall detection system. The proposed is used to measure the physical parameters like body temperature, heartbeat, Humidity and MEMS accelerometer monitoring with the help of sensors. Conventionally there are number of techniques available for the ICU patient's health monitoring system with wired communication technology. In the novel system the elderly person health is continuously monitored and the acquired data is transmitted to an PIC Microcontroller using Zigbee wireless sensor networks. Embedded processor supports for analyzing the input from the patient or elderly person and the results of all the parameters are stored in the database. If any abnormality felt by the patient automatic alarm sound will arrive. The implementation of the system is achieved by the advanced processor and simulation results are obtained by Keil c software.
\end{abstract}

Keywords: PIC16F877, sensors, Zigbee and Keil C.

\section{INTRODUCTION}

Recently wireless sensors and sensor networks plays a vital role in the research, technological community. But there are different from traditional wireless networks as well as computer networks, today the progress in science and technology offers miniature, speed, intelligence, sophistication, and new materials at lower cost, resulting in the development of various high-performance smart sensing system. Many new research is focused at improving quality of human life in terms of health by designing and fabricating sensors which are either in direct contact with the human body (invasive) or indirectly (noninvasive),

In the current proposed system the patient health is continuously monitored by the patient monitoring system and the acquired data is transmitted to a centralized PIC Microcontroller using Wireless Sensor Networks. A Zigbee node is connected to every elderly person monitor system that consumes very low power and is extremely small in size.

\section{LITERATURE SURVEY}

Priyadharshini et al was explains a novel idea to provide the information automatically to patients to take their right dosages at appropriate time. Now a day, most of the patients may forget to take their medicines as per the prescription due to mental stress. Hence, it may cause prolong period to recover from the diseases. Sometimes, the aged patients are gulping tablets and their dosage level incorrectly causing a severe problem. Henceforth, it is necessary to the patient to take proper medicines at precise quantity and time. To overcome the problems, a novel Automatic Medication Reminder (AMR) system is proposed [1].

Maradugu Anil Kumar et al was proposed a novel idea for continuous monitoring patient's health conditions. The health care scheme is focus on the measurement and monitoring various biological parameters of patient's body like heart rate, oxygen saturation level in blood and temperature using a web server and android application, where doctor can continuously monitor the patient's condition on his smart phone using an Android application. And also the patient history will be stored on the web server and doctor can access the information whenever needed from anywhere and need not physically present [2].

Gagana $\mathrm{P}$ et al was explains a novel idea when a person falls and is not able to get assistance within an hour, casualties arising from the fall can result in fatalities as early as 6 months later. It would seem then that a choice between safety and independence must be made. Fortunately, as health care technology advances, simple devices can be made to detect or even predict falls in the elderly, which could easily save lives without too much intrusion on their independence. Much research has been done on the topic of fall detection and fall prediction. Some have attempted to detect falls using a variety of sensors such as cameras, accelerometers, microphones, or a combination of the same. This paper is aimed at reporting which existing methods have been found effective and the combination of which will assist in the progression towards a safe, unobtrusive monitoring system for independent living.[3].

Malika et al was proposed a Wireless sensor network is a growing field showing tremendous applications in the areas of medical assistance in hospitals. According to the Medical Statistics everyday many patients' lives are affected due to negligence in the part of providing immediate care to the patients. Sometimes it is difficult for the staff in the hospitals to race against the time to reach the patient. Solution to this is a need of system that can measure patient health constantly. The main goal of this paper is to alert the staff in the hospitals in case of emergency. This system is designed using sensors and wireless technology with the help of Microcontroller. 
Sensors will continuously monitor the vital signs until an abnormal condition is detected. After detecting the abnormal condition, alert system comes into action which acts as a multi-alert system [4].

P.Karthick was explains this paper is to implement a prototype model for the real time patient monitoring system. The proposed is used to measure the physical parameters like body temperature, heartbeat, ECG, blood sugar, and oxygen level monitoring with the help of biosensors. Conventionally there are number of techniques available for the ICU patient's health monitoring system with wired communication technology.

In the novel system the patient health is continuously monitored and the acquired data is transmitted to an ARM server using zigbee wireless sensor networks. Embedded processor supports for analyzing the input from the patient and the results of all the parameters are stored in the database. If any abnormality felt by the patient automatic alarm sound will arrive and the message will send to the doctor mobile automatically by using GSM module. The implementation of the system is achieved by the advanced processor and simulation results are obtained by Keil c software [5].

Purnima et al was explains this paper is based on monitoring of patients. We have designed and developed a reliable, energy efficient patient monitoring system. It is able to send parameters of patient in real time. It enables the doctors to monitor patients' health parameters (temp, heartbeat, ECG, position) in real time. Here the parameters of patient are measured continuously (temp, heartbeat, ECG) and wirelessly transmitted using Zigbee. This project provides a solution for enhancing the reliability and flexibility by improving the performance and power management of the patient monitoring system. In the current proposed system the patient health is continuously monitored and the acquired data is analyzed at a centralized ARM microcontroller. If a particular patient's health parameter falls below the threshold value, an automated SMS is sent to the pre-configured Doctors mobile number using a standard GSM module interfaced to the ARM microcontroller. Here, we are using Zigbee for wireless transmission. The Doctor can get are cord of a particular patients information by just accessing the database of the patient on his PC which is continuously updated through Zigbee receiver module [6].

Manisha Shelar et al was explains this paper presents the development of a microcontroller based system for wireless heartbeat and temperature monitoring using ZigBee. In India many patients are dying because of heart attacks and reason behind that they are not getting timely and proper help. To give them timely and proper help first we want to continuous monitoring of patient health. The fixed monitoring system can be used only when the patient is on bed and this system are huge and only available in the hospitals in ICU. The system is developed for home use by patients that are not in a critical condition but need to be constant or periodically monitored by clinician or family. So that we can easily save many lives by providing them quick service [7].

\section{PROPOSED SYSTEM}

\section{TRANSMITTING SECTION:}

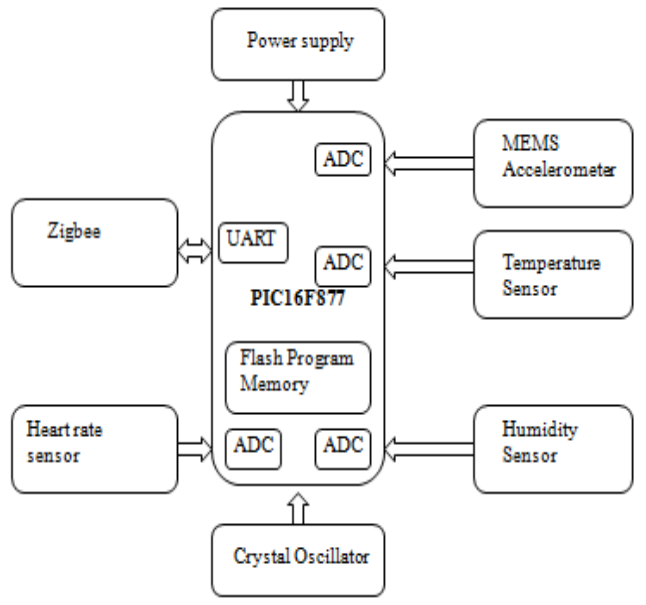

Fig-1 Transmitting Section

\section{RECEIVING SECTION:}

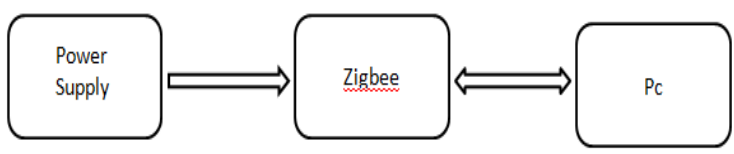

Fig-2 Receiver Section

\section{Working Principle:}

In Transmitter section of the monitoring system four sensors are used. These sensors are T: Temperature sensor, H: Humidity sensor, B: Heart beat sensor, M: MEMS sensor. All sensors are attached to Zigbee via PIC microcontroller as the brain of the system as explained in Figure 1 which is the block diagram of the system. Temperature sensors used here is LM35 which is a precise integrated-circuit sensor whose output voltage is linearly proportional to Celsius temperature. For Tri Axis MEMS Accelerometer used to sense the fall movements. The humidity sensor used here to provide comfort to asthmatic patients. Its function is to measure amount of water molecules in air. Finally the heart beat sensor to measure the heart rate. Then, these sensed data will go to Microcontroller PIC16F877 for further processing before transmit through Wireless Zigbee network. This combination of Sensors, PIC Microcontroller and Zigbee acknowledge as Transmitter part and will be placed near the elderly Person.

In receiver section of all the sensors, each of the sensors will continuously check for the data. Each node after obtaining the real time data will send the data to Zigbee via PIC microcontroller. In receiver part, the sensed real time data will be received by another Zigbee for analysis purpose. The Data received is sent to PIC16F877 for control action. Here limits are defined for each of the sensor node according to the requirements and can be change with the progress in patient. After checking for the 
limits, status of the patient under supervision will be known. If the data is more than the limits defined, status is Abnormal and if less than or equal to the limits defined, status is Normal.

\section{HARDWARE DESCRIPTION}

\section{A. ZigBee}

ZigBee is an IEEE802.15.4-based specification for a suite of high- level communication protocols used to create personal area networks with small, low-power digital radios. The technology defined by the ZigBee specification is intended to be simpler and less expensive than other wireless personal area networks (WPANs),such as Bluetooth or Wi-Fi. Its low power consumption limits transmission distances to 10-100 meters line-of-sight, depending on power output and environmental characteristics.

\section{B.PIC microcontroller}

PIC microcontrollers are a family of specialized microcontroller chips produced by Microchip Technology in Chandler, Arizona. The acronym PIC stands for "peripheral interface controller," although that term is rarely used nowadays. A microcontroller is a compact microcomputer designed to govern the operation of embedded systems in motor vehicles, robots, office machines, medical devices, mobile radios, vending machines, home appliances, and various other devices. A typical microcontroller includes a processor, memory, and peripheral.

\section{Heart Rate Sensor}

Heart beat sensor is designed to give digital output of heat beat when a finger is placed on it. When the eart beat detector is working, the beat LED flashes in unison with each heart beat. This digital output can be connected to microcontroller directly to measure the Beats Per Minute (BPM) rate. It works on the principle of light modulation by blood flow through finger at each pulse.

\section{MEMS Accelerometer}

Most accelerometers are Micro-Electro-Mechanical Sensors (MEMS). The basic principle of operation behind the MEMS accelerometer is the displacement of a small proof mass etched into the silicon surface of the integrated circuit and suspended by small beams.

\section{E. Humidity sensor}

A humidity sensor (or hygrometer) senses, measures and reports the relative humidity in the air. It therefore measures both moisture and air temperature. Relative humidity is the ratio of actual moisture in the air to the highest amount of moisture that can be held at that air temperature. The warmer the air temperature is, the more moisture it can hold.

\section{F. Temperature sensor}

A temperature sensor is a device, typically, a thermocouple or RTD,that provides for temperature measurement through an electrical signal. A thermocouple (T/C) is made from two dissimilar metals that generate electrical voltage in direct proportion to changes in temperature.

\section{SOFTWARE IMPLEMENTATION}

\section{A. Keil C $\mu$ VISION 4}

The $\mu$ Vision IDE from KEIL combines project management, make facilities source code editing, program debugging, and complete simulation in powerful environment. The $\mu$ Vision development platform is easy to use and helping you quickly creates embedded programs that work. The Keil C development tools for the ARM processor family support every level of developer from the professional applications engineer to the student just learning about embedded software development. The industry-standard Keil C Compilers, Macro Assemblers, Debuggers, Real-time Kernels, and Single-board Computers support all ARM processor compatible derivatives and help you get your projects completed on schedule. With the Keil tools, we can generate embedded applications for virtually every ARM derivative. The Keil Software ARM development tools are designed for the professional software developer; any level of programmer can use them to get the most output of the ARM processor architecture. Keil $\mathrm{C} \mu$ Vision 4 help provides the various simulation output.

\section{B. Algorithm}

Step 1: Start the process.

Step2: Initialize the sensors zigbee, $16 f 877$.

Step 3: Collect \& read data.

Step 4: Data transfer to the receiver through wireless communication.

Step 5: Checking for the status based on threshold value.

If threshold value is low, then normal.

If threshold value is high, then abnormal.

Threshold value is controlled based in PIC microcontroller.

Step 6: Stop the program.

C. Flow Chart

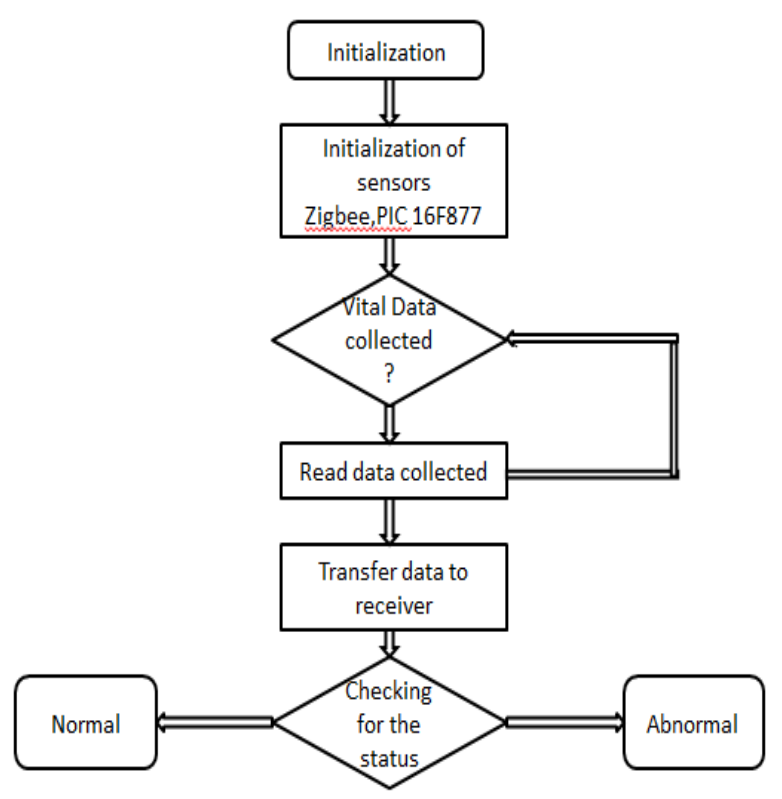

Fig-3 Flow chart for Proposed System 
INTERNATIONAL JOURNAL OF INNOVATIVE RESEARCH IN ELECTRICAL, ELECTRONICS, INSTRUMENTATION AND CONTROL ENGINEERING Vol. 4, Issue 2, February 2016

D. Simulation Results:
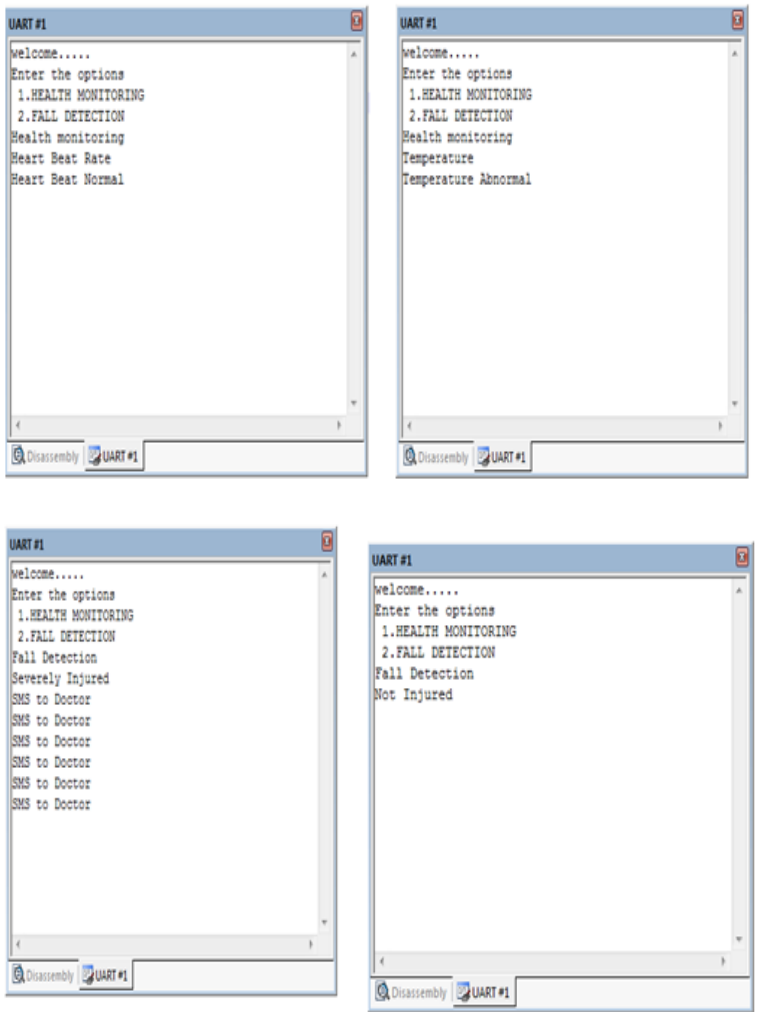

Fig-4 Representation of simulation results

\section{CONCLUSION}

In this proposed model of monitoring physiological parameters such as temperature, heartbeat, Humidity and MEMS Sensors are more powerful than currently available system. Currently available systems for monitoring physiological signals suffer from technical limitations. The proposed system is an enormous improvement over existing commercial methods; the present system can support more elderly persons with real-time, low-and results were discussed. In future we can expand this system by using Wi-Fi technology; through this technology we can monitor the more number of elderly peoples.

\section{REFERENCES}

[1] Priyadharshini. R, Ramya. S, Kalaiyarasi. S, Mithuna. S," A Novel Approach of Microcontroller based Automatic Medication Reminder (AMR) System for Patients", International Journal of Engineering Research \& Technology (IJERT), Vol. 4 Issue 04, April-2015.

[2] Maradugu Anil Kumar, Y.Ravi Sekhar, "Android Based Health Care Monitoring System", IEEE sponsored 2nd International Conference on Innovations in Information Embedded and Communication Systems ICIIECS'15.

[3] Gagana P, Vani K," Fall Detection Methods for the Safe and independent living of Elderly People and Patients", International Journal of Advanced Research in Computer and Communication Engineering, Vol. 4, Issue 3, March 2015.

[4] Malika, Charu Rana," A Multi-Alert Patient Health Monitoring using Zigbee", International Journal of Computer Applications (0975 - 8887) Volume 83 - No.17, December 2013.

[5] P. Karthick, C. Suresh Kumar, P. Arunprasad, S. Pushparaj, M. Jagadeeshraja N. Suthanthira Vanitha "Embedded Based Real-time
Patient Monitoring System", International Journal of VLSI and Embedded Systems-IJVES.

[6] Purnima, Neetu Rout, Rahul Tiwary ,Renuka Bhandari "Zigbee and GSM based Patient Health Monitoring System", International Journal of Advanced Research in Electrical, Electronics and Instrumentation Engineering (An ISO 3297: 2007 Certified Organization) Vol. 3, Issue 1, January 2014

[7] Manisha Shelar, Jaykaran Singh, Mukesh Tiwari "Wireless Patient Health Monitoring System", International Journal of Computer Applications (0975 -8887) Volume 62- No.6, January 2013. 